Pacific Journal of Mathematics

A GEOMETRIC CHARACTERIZATION OF INDETERMINATE 


\section{A GEOMETRIC CHARACTERIZATION OF INDETERMINATE MOMENT SEQUENCES}

E. P. Merkes and Marion Wetzel

Hamburger and Stieltjes moment sequences are studied from the standpoint of the geometry of their moment spaces. Necessary and sufficient conditions are obtained that each of these sequences be indeterminate. The elements in the associated Jacobi and Stieltjes type continued fractions are characterized in terms of ratios of distances in the moment spaces.

1. Introduction. A sequence of real numbers $\left\{c_{n}\right\}_{n=0}^{\infty}$ is an $H$ (Hamburger moment) sequence if there exists a bounded nondecreasing function $\gamma$ on $(-\infty, \infty)$ such that

$$
c_{n}=\int_{-\infty}^{\infty} t^{n} d \gamma(t) \quad(n=0,1,2, \cdots) .
$$

The function $\gamma$, called a mass function for the sequence $\left\{c_{n}\right\}$, is normalized to be left continuous and such that $\gamma(0)=0$. The sequence $\left\{c_{n}\right\}_{n=0}^{\infty}$ is an $S$ (Stieltjes moment) sequence if it is an $H$ sequence and there is a mass function $\gamma$ for the sequence that is constant on $(-\infty, 0)$. An $H$ sequence or an $S$ sequence is determinate if the mass function $\gamma$ for the sequence is unique. Otherwise the moment sequence is indeterminate.

The geometric approach of Carathéodory [2] for the classical moment problems has been extended and generalized by a number of authors (see [5]). In particular, Krein [6] initiated a geometric study of general Tchebycheff systems and Karlin and Shapley [4] rekindled interest in the geometry of moment sequences by their definitive memoir on the finite (Hausdorff) moment problem. The primary purpose of this paper is to provide, in the spirit of the works of Krein and of Karlin and Shapley, geometric characterizations for indeterminate $H$ sequences and for indeterminate $S$ sequences.

More specifically, let $\mathfrak{M}_{2 m+1}$ denote the set of vectors $c=$ $\left(c_{0}, c_{1}, \cdots, c_{2 m}\right)$ in Euclidean $E^{2 m+1}$ space such that there is a mass function $\gamma$ on $(-\infty, \infty)$ for which (1) holds when $n=0,1,2, \cdots, 2 m$, For real $\lambda>0$ and for $c, c^{*}$ in $\mathfrak{M}_{2 m+1}$, the vectors $\lambda c$ and $c+c^{*}$ are also in $\mathfrak{M}_{2 m+1}$. Thus $\mathfrak{M}_{2 m+1}$ is a convex cone in $E^{2 m+1}$. For a given $c=\left(c_{0}, c_{1}, \cdots, c_{2 m}\right) \in \mathfrak{M}_{2 m+1}$, we consider the two dimensional 
section of this cone defined by

$$
D_{m}=\left\{(x, y):\left(x, y, c_{2}, c_{3}, \cdots, c_{2 m}\right) \in \mathfrak{M}_{2 m+1}\right\} .
$$

In $\S 3$ we prove that $D_{m}$ is a closed region bounded by a parabola (possibly degenerate) and that $D_{m} \subset D_{m-1}$. The intersection $\bigcap_{m=1}^{\infty} D_{m}$, called the limit parabolic region, is either a closed region containing $\left(c_{0}, c_{1}\right)$ bounded by a proper parabola or a ray $\left\{(x, y): x \geqq \underline{c}_{0}, y=c_{1}\right\}$ where $\underline{c}_{0} \leqq c_{0}$. An $H$ sequence is proved to be indeterminate if and only if the point $\left(c_{0}, c_{1}\right)$ is an interior point of the limit parabolic region. For an $S$ sequence there is a convex cone $\mathfrak{N}_{2 m+1}$ corresponding to $\mathfrak{M}_{2 m+1}$ and two dimensional sections $E_{m}^{\prime}$ of this cone are introduced in the same fashion as the sections $D_{m}$ were defined from $\mathfrak{M}_{2 m+1}$. An $S$ sequence is proved to be indeterminate if and only if $\left(c_{0}, c_{1}\right)$ is an interior point of $\bigcap_{m=1}^{\infty} E_{m}^{\prime}$ in $\S 4$. The final section of this paper provides a geometric interpretation of the coefficients of the $J$-fraction or $S$-fraction corresponding to $\sum_{m=0}^{\infty} c_{m} / z^{m+1}$ when $\left\{c_{m}\right\}_{m=0}^{\infty}$, is an $H$ or an $S$ sequence respectively.

2. Preliminaries. For a real sequence $\left\{c_{n}\right\}_{n=0}^{\infty}$ let

$$
\Delta_{n, p}=\left|\begin{array}{cccc}
c_{p} & c_{p+1} & \cdots & c_{p+n} \\
c_{p+1} & c_{p+2} & \cdots & c_{p+n+1} \\
\vdots & \vdots & & \vdots \\
c_{p+n} & c_{p+n+1} & \cdots & c_{p+2 n}
\end{array}\right| \quad(n, p=0,1,2, \cdots) .
$$

For brevity set $\Delta_{n}=\Delta_{n, 0}$. A classical necessary and sufficient condition for $\left\{c_{n}\right\}_{n=0}^{\infty}$ to be an $H$ sequence is that either (a) $\Delta_{n}>0$ for $n=0$, $1,2, \cdots$ or (b) $\Delta_{n}>0$ for $n=0,1,2, \cdots, m-1$ and $\Delta_{n}=0$ for $n=$ $m, m+1, \cdots([3],[7$, p. 5]). An $H$ sequence is called positive definite or positive semidefinite according as (a) or (b) holds. A positive semidefinite $H$ sequence is always determinate. A positive definite $H$ sequence is determinate if and only if at least one of the sequences

$$
\left\{\Delta_{n} / \Delta_{n-1,2}\right\}, \quad\left\{\Delta_{n, 2} / \Delta_{n-1,4}\right\}
$$

has limit zero as $n \rightarrow \infty$ ([3] [7, p. 72]). Let

$$
\frac{a_{0}^{2}}{z+b_{1}}-\frac{a_{1}^{2}}{z+b_{2}}-\cdots-\frac{a_{n}^{2}}{z+b_{n+1}}-\cdots
$$

be the $J$-fraction expansion of the formal power series $\sum_{n=0}^{\infty} c_{n} / z^{n+1}$. If $a_{j} \neq 0(j=0,1,2, \cdots, n)$, let

$$
P_{n}^{*}(z)=\frac{B_{n}(z)}{a_{0} a_{1} \cdots a_{n}}, \quad Q_{n}^{*}(z)=\frac{A_{n}(z)}{a_{0} a_{1} \cdots a_{n}},
$$


where $B_{n}(z)$ and $A_{n}(z)$ respectively denote the $n$th denominator and $n$th numerator of the continued fraction (3). Since $\Delta_{n}=a_{0}^{2} a_{1}^{2} \cdots a_{n}^{2} \Delta_{n-1}$ [8, p. 197], the polynomials $P_{n}^{*}, Q_{n}^{*}$ are defined for $n \leqq m$ if $\Delta_{n} \neq 0$, $n \leqq m$. When $\Delta_{n} \neq 0, n \leqq m$, we have furthermore that

$$
\begin{gathered}
F_{m}^{*}=\sum_{j=0}^{m}\left[P_{j}^{*}(0)\right]^{2}=-\frac{1}{\Delta_{m}}\left|\begin{array}{ccccc}
0 & 1 & 0 & \cdots & 0 \\
1 & c_{0} & c_{1} & \cdots & c_{m} \\
0 & c_{1} & c_{2} & \cdots & c_{m+1} \\
\vdots & \vdots & \vdots & & \vdots \\
0 & c_{m} & c_{m+1} & \cdots & c_{2 m}
\end{array}\right|, \\
G_{m}^{*}=\sum_{j=0}^{m} P_{j}^{*}(0) Q_{j}^{*}(0)=-\frac{1}{\Delta_{m}}\left|\begin{array}{ccccc}
0 & 0 & c_{0} & \cdots & c_{m-1} \\
1 & c_{0} & c_{1} & \cdots & c_{m} \\
0 & c_{1} & c_{2} & \cdots & c_{m+1} \\
\vdots & \vdots & \vdots & & \vdots \\
0 & c_{m} & c_{m+1} & \cdots & c_{2 m}
\end{array}\right|,
\end{gathered}
$$

and

$$
H_{m}^{*}=\sum_{j=0}^{m}\left[Q_{j}^{*}(0)\right]^{2}=-\frac{1}{\Delta_{m}}\left|\begin{array}{lllll}
0 & 0 & c_{0} & \cdots & c_{m-1} \\
0 & c_{0} & c_{1} & \cdots & c_{m} \\
c_{0} & c_{1} & c_{2} & \cdots & c_{m+1} \\
\vdots & \vdots & \vdots & & \vdots \\
c_{m-1} & c_{m} & c_{m+1} & \cdots & c_{2 m}
\end{array}\right|
$$

(See, for example, [9].)

Set $\Delta_{-1,1}=1$ and assume $c_{0}>0$. Then a necessary and sufficient condition for $\left\{c_{n}\right\}_{n=0}^{\infty}$ to be an $S$ sequence is that either (c) $\Delta_{n}>0$ and $\Delta_{n, 1}>0$ for $n=0,1,2, \cdots$ or (d) $\Delta_{n}>0$ and $\Delta_{n-1,1}>0$ for $n=$ $0,1,2, \cdots, m ; \Delta_{m, 1} \geqq 0 ;$ and $\Delta_{n}=\Delta_{n, 1}=0$ for $n=m+1, m+2, \cdots$ [7, p. 6]. The polynomials $P_{n}^{*}, Q_{n}^{*}$ can be defined for an $S$ sequence by (4) where the continued fraction (3) is the even part of the $S$ fraction

$$
\frac{d_{0}}{z}-\frac{d_{1}}{1}-\frac{d_{2}}{z}-\frac{d_{3}}{1}-\cdots-\frac{d_{2 n}}{z}-\frac{d_{2 n+1}}{1}-\cdots
$$

corresponding to the formal power series $\sum_{j=0}^{\infty} c_{j} / z^{j+1}[8$, p. 73].

3. $H$ sequences. Let $\left(c_{0}, c_{1}, c_{2}, \cdots, c_{2 m}\right) \in \mathfrak{M}_{2 m+1}$. For real $x$ and $y$, the vector $\left(x, y, c_{2}, c_{3}, \cdots, c_{2 m}\right) \in \mathfrak{M}_{2 m+1}$ if and only if 
(9) $\quad D_{n}(x, y)=\left|\begin{array}{ccccc}x & y & c_{2} & \cdots & c_{n} \\ y & c_{2} & c_{3} & \cdots & c_{n+1} \\ c_{2} & c_{3} & c_{4} & \cdots & c_{n+2} \\ \vdots & \vdots & \vdots & \vdots \\ c_{n} & c_{n+1} & c_{n+2} & \cdots & c_{2 n}\end{array}\right| \geqq 0 \quad(n=1,2, \cdots, m)$.

Suppose $A_{n}>0$ for $n<m$. Then by (5), (6), and (7) with each $c_{j}$ replaced by $c_{j+2}$ we have

$$
D_{n}(x, y)=\Delta_{n-1,2}\left\{x-\sum_{j=0}^{n-1}\left[Q_{j}(0)+y P_{j}(0)\right]^{2}\right\},
$$

where $P_{j}(z), Q_{j}(z)$ are the polynomials (4) for the sequence $c_{2}, c_{3}, \cdots$, $c_{2 m}$. Since $\Delta_{n}>0$ implies $\Delta_{n-1,2}>0$ for $0<n<m$, the set $D_{n}$ of points $(x, y)$ such that $D_{n}(x, y) \geqq 0$ is a closed convex region bounded by a parabola. By (10) $D_{n} \subset D_{n-1}, 1<n<m$, and the boundaries of $D_{n}$ and of $D_{n-1}$ have exactly one point (possibly infinity) in common.

LEMMA 1. Let $\left\{c_{n}\right\}_{n=0}^{\infty}$ be a positive definite $H$ sequence. For each positive integer $n$, the two dimensional section

$$
D_{n}=\left\{(x, y):\left(x, y, c_{2}, c_{3}, \cdots, c_{2 m}\right) \in \mathfrak{M}_{2 m+1}\right\}
$$

is a closed convex region bounded by the parabola

$$
x-x_{n}=F_{n}\left(y-y_{n}\right)^{2},
$$

where $x_{n}=H_{n}-G_{n}^{2} / F_{n}, y_{n}=-G_{n} / F_{n}$ and

$$
F_{n}=\sum_{j=0}^{n}\left[P_{j}(0)\right]^{2}, G_{n}=\sum_{j=0}^{n} P_{j}(0) Q_{j}(0), H_{n}=\sum_{j=0}^{n}\left[Q_{j}(0)\right]^{2} .
$$

Furthermore, $D_{n} \subset D_{n-1}, \bigcap_{n=1}^{\infty} D_{n} \neq \varnothing$, and the sequences $\left\{x_{n}\right\},\left\{y_{n}\right\}$ converge to finite limits.

Proof. The boundary (12) is obtained from (5), (6), and (7) when the indices are augmented by 2 by a straight-forward calculation (see also [9]). The fact that the parabolic regions $D_{n}$ are nested follows immediately from (9) and (10). One consequence of these observations is that the sequence $\left\{x_{n}\right\}$ is nondecreasing. Since $\left(c_{0}, c_{1}\right) \in D_{n}$ for all $n$, we have $x_{n} \leqq c_{0}$ and, hence, $\lim _{n \rightarrow \infty} x_{n} \leqq c_{0}$. To prove $\left\{y_{n}\right\}$ also converges, first note that $\left\{F_{n}\right\}$ is a nondecreasing sequence of positive numbers. Hence $F_{n}$ tends to a positive limit or $\infty$ as $n \rightarrow \infty$. From the nesting of the regions, we have $\left(x_{m}, y_{m}\right) \in D_{n}$ whenever $m \geqq n$. By (12) this implies

$$
\left(x_{m}-x_{n}\right) / F_{n} \geqq\left(y_{m}-y_{n}\right)^{2} .
$$


It follows that $\left\{y_{n}\right\}$ tends to a finite limit as $n \rightarrow \infty$.

Let $x^{0}=\lim x_{n}, y^{0}=\lim y_{n}(n \rightarrow \infty)$ under the hypothesis of Lemma 1. If $F_{n} \rightarrow F<\infty$, then $\bigcap_{n=1}^{\infty} D_{n}$ is a closed region bounded by the parabola $x-x^{0}=F\left(y-y^{0}\right)^{2}$. If $F_{n} \rightarrow \infty$, then the length of the latus rectum $1 / F_{n}$ of the $n$th parabola (12) tends to zero as $n \rightarrow \infty$. In this case $\bigcap_{n=1}^{\infty} D_{n}$ is a ray: $x \geqq x^{0}, y=y^{0}$.

When $\Delta_{n}>0$ for $n<m$, we have $\Delta_{n-1,2}>0$ and we define $\underline{c}_{0, n}$ by

$$
\left|\begin{array}{llll}
\underline{c}_{0, n} & c_{1} & \cdots & c_{n} \\
c_{1} & c_{2} & \cdots & c_{n+1} \\
\vdots & \vdots & & \vdots \\
c_{n} & c_{n+1} & \cdots & c_{2 n}
\end{array}\right|=0 .
$$

Then $c_{0}-\underline{c}_{0, n}=\Delta_{n} / \Delta_{n-1,2}$ so $c_{0}>\underline{c}_{0, n}, n<m$. The projection $\left(\underline{c}_{0, n}, c_{1}\right.$, $\left.\cdots, c_{2 n}\right)$ is a boundary point of $\mathfrak{M}_{2 n+1}$.

LEMMA 2. Let $\left\{c_{n}\right\}_{n=0}^{\infty}$ be a positive semidefinite $H$ sequence for which $\Delta_{n}>0$ when $n<m$ and $\Delta_{n}=0$ when $n \geqq m$. If $\Delta_{m-1,2}>0$, then the $m$ th region (11) is bounded by a proper parabola that contains the point $\left(c_{0}, c_{1}\right)$. The $m+1$ st region (11) degenerates in this case to a ray $x \geqq c_{0}, y=c_{1}$. If $\Delta_{m-1,2}=0$, the $m$ th region (11) is a ray $x \geqq \underline{c}_{0, m-1}, y=c_{1}$.

Proof. Suppose $\Delta_{m-1,2}>0$. Then $\Delta_{m-2,4}>0$ and by (9) the length of the latus rectum $\Delta_{m-1,2} / \Delta_{m-2,4}$ of the $m$ th parabola $D_{m}(x, y)=0$ is not zero. Furthermore $D_{m}\left(c_{0}, c_{1}\right)=\Delta_{m}=0$ so $\left(c_{0}, c_{1}\right)$ is on the $m$ th parabola. We can define $\underline{c}_{0, m}$ by $(14)$ in this case and $\underline{c}_{0, m}=c_{0}$. We show next that the $m+1$ st parabolic region $D_{n}(x, y) \geqq 0(n=$ $1,2, \cdots, m+1)$ is a ray $x \geqq c_{0}, y=c_{1}$ when $\Delta_{m-1,2}>0$. Since $\Delta_{n}>0$, $n<m$ and $\Delta_{n}=0, n \geqq m$, the moment problem for the sequence $\left\{c_{n}\right\}$ is determinate. This implies there is a unique representation

$$
c_{n}=\sum_{j=1}^{m} \lambda_{j} t_{j}^{n} \quad(n=0,1,2, \cdots),
$$

where $\lambda_{j}>0(j=1,2, \cdots, m)$ and $t_{1}<t_{2}<\cdots<t_{m}$. Since $\underline{c}_{0, m}=c_{0}$, we have $t_{j} \neq 0(j=1,2, \cdots, m)$. By replacing each $c_{n}$ in $A_{m, 2}$ with its representation (15), we can express the determinant $\Delta_{m, 2}$ as a linear combination of determinants of the form

$$
\left|\begin{array}{llll}
1 & 1 & \cdots & 1 \\
t_{k_{1}} & t_{k_{2}} & \cdots & t_{k_{m+1}} \\
\vdots & \vdots & & \vdots \\
t_{k_{1}}^{m} & t_{k_{2}}^{m} & \cdots & t_{k_{m+1}}^{m}
\end{array}\right|,
$$


where the indices $k_{j}$ are between 1 and $m$. Since each of these last determinants is of order $m+1$, each contains at least two identical columns. We conclude that $\Delta_{m, 2}=0$ and, therefore, $D_{m+1}(x, y)$ is independent of $x$. Now $D_{m+1}(x, y) \not \equiv 0$ since the coefficient of $y^{2}$ is $\Delta_{m-1,4} \neq 0$. To prove this last assertion, assume $\Delta_{m-1,4}=0$. This implies there is a representation

$$
c_{n+4}=\sum_{j=1}^{p} \mu_{j} s_{j}^{n} \quad(n=0,1,2, \cdots),
$$

where $s_{1}<s_{2}<\cdots<s_{p}, \mu_{j}>0(j=1,2, \cdots, p), p \leqq m-1$. Since $t_{j} \neq 0(j=1,2, \cdots, m)$ in (15), there are two distinct representations, (15) and (16), for the semidefinite $H$ sequence $\left\{c_{n}\right\}_{n=4}^{\infty}$. This is contrary to the fact that this sequence is determinate and, thus, $\Delta_{m-1,4} \neq 0$. Finally $D_{m+1}(x, y)=0$ has a unique double root at $y=c_{1}$ by known results on symmetric determinants [1, p. 139].

Next assume $\Delta_{m-1,2}=0$. This implies $D_{m}(x, y)$ is independent of $x$ and, as in the previous case, we conclude $D_{m}(x, y)=-\Delta_{m-2,4}\left(y-c_{1}\right)^{2}$. Since $D_{m-1}(x, y) \geqq 0$ forces $x$ to be not smaller than $\underline{c}_{0, m-1}$ when $y=c_{1}$, we have $D_{m}=\left\{(x, y): x \geqq \underline{c}_{0, m-1}, y=c_{1}\right\}$. This completes the proof of the Lemma.

Using the representation (15) we can easily prove $D_{m+j}(x, y)=0$ for all choices of $(x, y)$ when $j \geqq 2$ and $\left\{c_{n}\right\}_{n=0}^{\infty}$ is a positive semidefinite $H$ sequence with $\Delta_{m-1}>0, \Delta_{m}=0$. This means that the conditions $D_{n}(x, y) \geqq 0(n=1,2, \cdots)$ add no new restrictions on $(x, y)$ once $n>m+1$, that is, once the parabolic regions degenerate to a ray. It is meaningful, therefore, to speak of this ray as the limit region $\bigcap_{n=1}^{\infty} D_{n}$ for a positive semidefinite $H$ sequence.

THEOREM 1. Let $\left\{c_{n}\right\}_{n=0}^{\infty}$ be an $H$ sequence. This sequence is indeterminate if and only if $\left(c_{0}, c_{1}\right)$ is an interior point of the limit parabolic region.

Proof. If $\left\{c_{n}\right\}$ is positive semidefinite, then the limit region is a ray by Lemma 2. The limit region has no interior points in this case and the sequence is determinate.

Suppose $\left\{c_{n}\right\}$ is positive definite. By (5) and (12) the length of the latus rectum of the $n$th parabola is $1 / F_{n}=\Delta_{n-1,2} / \Delta_{n-2,4}$. If $\lim \Delta_{n-1,2} / \Delta_{n-2,4}=0$ as $n \rightarrow \infty$, then the limit parabolic region is a ray and by (2) the sequence is determinate. Suppose therefore $\Delta_{n-1,2} / \Delta_{n-2,4}$ has a nonzero limit as $n \rightarrow \infty$. By (14) we have $\left(\underline{c}_{0, n}, c_{1}\right)$ is on the $n$th parabola and $c_{0}-\underline{c}_{0, n}=\Delta_{n} / \Delta_{n-1,2}>0$. Since the parabolas are nested by Lemma 1 , the sequence $\left\{\underline{c}_{0},{ }_{n}\right\}$ is nondecreasing. Let $\underline{c}_{0, n} \rightarrow c_{0}$ as $n \rightarrow \infty$. Then $\underline{c}_{0} \leqq c_{0}$ and equality holds if and only if $\Delta_{n} / \Delta_{n-1,2} \rightarrow 0$ 
as $n \rightarrow \infty$. The last condition implies $\left\{c_{n}\right\}$ is determinate by (2). Furthermore, $c_{0}=\underline{c}_{0}$ implies $\left(c_{0}, c_{1}\right)$ is on the boundary of the limit parabolic region. Now if $\underline{c}_{0}<c_{0}$, then $\left(c_{0}, c_{1}\right)$ is interior to the limit parabolic region and by (2) the sequence $\left\{c_{n}\right\}$ is indeterminate.

4. $S$ sequences. Every $S$ sequence is an $H$ sequence so the existence of the limit parabolic region is assured by Lemma 1 and Lemma 2. Furthermore a sequence $\left\{c_{n}\right\}_{n=0}^{\infty}$ is an indeterminate $S$ sequence if and only if $\left\{p_{n}\right\}_{n=0}^{\infty}$, where $p_{2 n}=c_{n}, p_{2 n+1}=0(n=0,1,2, \cdots)$, is an indeterminate $H$ sequence. Replacing $\left\{c_{n}\right\}$ by $\left\{p_{n}\right\}$ in (2), we obtain the following criteria for an indeterminate $S$ sequence.

LEMMA 3. An S sequence is indeterminate if and only if the sequences $\left\{\Delta_{n} / \Delta_{n-1,2}\right\}$ and $\left\{\Delta_{n, 1} / \Delta_{n-1,3}\right\}$ each have nonzero limits as $n \rightarrow \infty$.

The following determinant identity is needed.

LEMMA 4. $\Delta_{n-1,2} \Delta_{n-1}-\Delta_{n-2,2} \Delta_{n}=\Delta_{n-1,1}^{2}, n \geqq 1,\left(\Delta_{-1,2}=1\right)$.

Proof. For a sequence $\left\{c_{j}\right\}_{j=0}^{2 n+1}$ set

$$
\Delta_{n}(t)=\left|\begin{array}{cccc}
1 & t & \cdots & t^{n} \\
c_{1} & c_{2} & \cdots & c_{n+1} \\
\vdots & \vdots & \vdots \\
c_{n} & c_{n+1} & \cdots & c_{2 n}
\end{array}\right|=(-1)^{n} \Delta_{n-1,1} t^{n}+\cdots+\Delta_{n-1,2} \cdot
$$

Define the linear functional $M$ on the linear space of polynomials of degree not exceeding $2 n+1$ by the condition $M\left[t^{j}\right]=c_{j}(j=$ $0,1,2, \cdots, 2 n+1)$. Using elementary properties of determinants, we have

$$
M\left[t^{j} \Delta_{n}(t)\right]= \begin{cases}\Delta_{n} & \text { if } j=0 \\ 0 \quad \text { if } & 0<j \leqq n \\ (-1)^{n} \Delta_{n, 1} & \text { if } j=n+1 .\end{cases}
$$

Therefore,

$$
\begin{aligned}
M\left[\Delta_{n-1}(t) \Delta_{n}(t)\right] & =M\left[\left\{(-1)^{n-1} \Delta_{n-2,1} t^{n-1}+\cdots+\Delta_{n-2,2}\right\} \Delta_{n}(t)\right] \\
& =\Delta_{n-2,2} \Delta_{n}
\end{aligned}
$$

and

$$
\begin{aligned}
M\left[\Delta_{n-1}(t) \Delta_{n}(t)\right] & =M\left[\Delta_{n-1}(t)\left\{(-1)^{n} \Delta_{n-1,1} t^{n}+\cdots+\Delta_{n-1,2}\right\}\right] \\
& =(-1)^{n} \Delta_{n-1,1} M\left[t^{n} \Delta_{n-1}(t)\right]+\Delta_{n-1,2} M\left[\Delta_{n-1}(t)\right] \\
& =-\Delta_{n-1,1}^{2}+\Delta_{n-1,2} \Delta_{n-1} .
\end{aligned}
$$


The identity follows from these equalities.

Let $\left\{c_{n}\right\}_{n=0}^{\infty}$ be an $S$ sequence such that $\Delta_{n, 1}>0$ for $n \leqq m$. We define $\underline{c}_{1, m}$ by

$$
\left|\begin{array}{llll}
\underline{c}_{1, m} & c_{2} & \cdots & c_{m+1} \\
c_{2} & c_{3} & \cdots & c_{m+2} \\
\vdots & \vdots & \vdots \\
c_{m+1} & c_{m+2} & \cdots & c_{2 m+1}
\end{array}\right|=0
$$

Since $\Delta_{m-1,3}>0$ in this case, we have $c_{1}-\underline{c}_{1, m}=\Delta_{m, 1} / \Delta_{m-1,3}$ and $c_{1}>\underline{c}_{1, m}$, If $\Delta_{m+1,1}=0$, define $\underline{c}_{1, m+1}$ to be $c_{1}$.

LEMMA 5. Let $\left\{c_{n}\right\}_{n=0}^{\infty}$ be an $S$ sequence. The sequence $\left\{\underline{c}_{1, n}\right\}$ is nondecreasing and $\underline{c}_{1}=\lim \underline{c}_{1, n} \leqq c_{1}$.

Proof. Since $\underline{c}_{1, n}$ equals $c_{1}$ from some index $n$ onward when $\left\{\underline{c}_{n}\right\}$ is positive semidefinite, we assume $\Delta_{n}>0, \Delta_{n, 1}>0$ for $n \leqq m$. Then

$$
\underline{c}_{1, m}-\underline{c}_{1, m-1}=\frac{\Delta_{m-1,1}}{\Delta_{m-2,3}}-\frac{\Delta_{m, 1}}{\Delta_{m-1,3}}=\frac{\Delta_{m-1,2}^{2}}{\Delta_{m-2,3} \Delta_{m-1,3}},
$$

where the last equality is obtained by the identity of Lemma 4 applied to the sequence $\left\{c_{n}\right\}_{n=1}^{\infty}$. It follows that $\left\{\underline{c}_{1, n}\right\}$ is is nondecreasing and bounded by $c_{1}$.

Let $\mathfrak{R}_{2 m+1}$ denote the subset of $\mathfrak{M}_{2 m+1}$ consisting of $S$ sequences. If $\left\{c_{n}\right\}_{n=0}^{\infty}$ is an $S$ sequence and $\Delta_{n}>0, \Delta_{n, 1}>0$ for $n \leqq m$, then the vector $\left(x, y, c_{2}, \cdots, c_{2 m}\right) \in \mathfrak{N}_{2 m+1}$ if and only if $(x, y) \in D_{m}$ and $y \geqq \underline{c}_{1, m}$. Since $\underline{c}_{1, m} \rightarrow \underline{c}_{1}$ as $m \rightarrow \infty$, the limit region corresponding to an $S$ sequence is either a ray $x \geqq \underline{c}_{0}, y=c_{1}=\underline{c}_{1}$ or the intersection of a proper limit parabolic region and the half plane $y \geqq \underline{c}_{1}$.

THEOREM 2. Let $\left\{c_{n}\right\}_{n=0}^{\infty}$ be an $S$ sequence. Then $\left\{c_{n}\right\}$ is indeterminate if and only if $\left(c_{0}, c_{1}\right)$ is interior to the limit parabolic region and $c_{1}>\underline{c}_{1}$.

Proof. If $\left\{c_{n}\right\}_{n=0}^{\infty}$ is positive semidefinite, the sequence is determinate and the limit parabolic region has no interior points. Suppose, therefore, $\left\{c_{n}\right\}$ is positive definite. If the limit parabolic region is a ray $x \geqq \underline{c}_{0}, y=c_{1}$, then $c_{1}=\underline{c}_{1}$ and, hence, $\Delta_{n, 1} / \Delta_{n-1,3} \rightarrow 0$ as $n \rightarrow \infty$. The sequence $\left\{c_{n}\right\}$ is determinate in this case by Lemma 3 . If the limit parabolic region has interior points, then $\left(c_{0}, c_{1}\right)$ is on its boundary if and only if $c_{0}-\underline{c}_{0, n}=\Delta_{n} / \Delta_{n-1,2} \rightarrow 0$ as $n \rightarrow \infty$. Again by Lemma 3 , the sequence $\left\{c_{n}\right\}$ is determinate when the last condition holds. Finally let $\left(c_{0}, c_{1}\right)$ be an interior point of the limit parabolic region. 
Then $\left\{\Delta_{n} / \Delta_{n-1,2}\right\}$ does not tend to zero as $n \rightarrow \infty$. In this case, $\left\{c_{n}\right\}$ is determinate if and only if $c_{1}-\underline{c}_{1, n}=\Delta_{n, 1} / \Delta_{n-1,3} \rightarrow 0$ as $n \rightarrow \infty$ by Lemma 3. The last condition is equivalent to the condition $c_{1}=\underline{c}_{1}$ and the proof is complete.

Corollary. Let $\left\{c_{n}\right\}_{n=0}^{\infty}$ be a positive definite $S$ sequence. This sequence is a determinate $S$ sequence and an indeterminate $H$ sequence if and only if $\left(c_{0}, c_{1}\right)$ is an interior point of the limit parabolic region and $c_{1}=\underline{c}_{1}=\lim _{n \rightarrow \infty} \underline{c}_{1, n}$.

5. A geometric interpretation of the continued fraction coefficients. For $n \geqq 1$, let $\Delta_{n-1}^{*}$ denote the minor of the element $c_{2 n-1}$ in the determinant $\Delta_{n}$. From the algorithm for expanding a power series into a $J$-fraction [8, p. 196] it is easily proved that the coeffcients of (3) are determined by

$$
a_{n}=\frac{\sqrt{\Delta_{n-2} \Delta_{n}}}{\Delta_{n-1}}, b_{n+1}=\frac{\Delta_{n-1}^{*} \Delta_{n}-\Delta_{n-1} \Delta_{n}^{*}}{\Delta_{n-1} \Delta_{n}}(0 \leqq n \leqq m),
$$

where $\Delta_{-1}^{*}=0, \Delta_{-1}=\Delta_{-2}=1$, and $\left\{c_{n}\right\}_{n=0}^{\infty}$ is an $H$ sequence such that $\Delta_{n}>0$ when $n \leqq m$. To obtain a geometric interpretation of these coefficients, we introduce the two dimensional sections of the cone $\mathfrak{M}_{2 n+1}$ defined by

$$
E_{n}=\left\{(x, y):\left(c_{0}, c_{1}, \cdots, c_{2 n-2}, x, y\right) \in \mathfrak{M}_{2 n+1}\right\}(n \leqq m) .
$$

Note that $E_{n}$ is a closed region bounded by the parabola

$$
\left|\begin{array}{lllll}
c_{0} & c_{1} & \cdots & c_{n-1} & c_{n} \\
c_{1} & c_{2} & \cdots & c_{n} & c_{n+1} \\
\vdots & \vdots & & \vdots & \vdots \\
c_{n-1} & c_{n} & \cdots & c_{2 n-2} x \\
c_{n} & c_{n+1} & \cdots & x & y
\end{array}\right|=0
$$

Let $\underline{c}_{2 n}$ denote the $y$-coordinate of the point on the parabola for which $x=c_{2 n-1}$. A simple computation proves that the axis of this parabola is $x=c_{2 n-1}^{*}$, where $\Delta_{2 n-1}^{*}$ is obtained by replacing $c_{2 n-1}$ in $\Delta_{n-1}^{*}$ with $c_{2 n-1}^{*}$ and setting the resulting determinant equal to zero.

THEOREM 3. Let $\left\{c_{n}\right\}_{n=0}^{\infty}$ be an $H$ sequence and let $\Delta_{n}>0$ for $n \leqq m$. Let $e_{n}$ and $e_{n}^{*}$ respectively denote the distance in the vertical direction from $\left(c_{2 n-1}, c_{2 n}\right)$ to the boundary of $E_{n}$ and the distance in the horizontal direction from $\left(c_{2 n-1}, c_{2 n}\right)$ to the axis of the parabolic boundary of $E_{n}$. Then the coefficients in (3) are 


$$
\begin{aligned}
a_{n}^{2}= & \frac{e_{n}}{e_{n-1}}, b_{n+1}=\frac{e_{n}^{*}}{e_{n-1}}-\frac{e_{n+1}^{*}}{e_{n}} \\
& \left(e_{0}^{*}=0, e_{-1}=1, e_{0}=c_{0}, 0 \leqq n \leqq m\right) .
\end{aligned}
$$

Furthermore $e_{n}=c_{2 n}-\underline{c}_{2 n}, e_{n+1}^{*}=c_{2 n+1}-c_{2 n+1}^{*}$ for $0 \leqq n \leqq m$.

Proof. From the definitions of $c_{2 n}$ and $\underline{c}_{2 n+1}^{*}$, we have for $0 \leqq$ $n \leqq m, e_{n}=c_{2 n}-\underline{c}_{2 n}=\Delta_{n} / \Delta_{n-1}, e_{n+1}^{*}=c_{2 n+1}-c_{2 n+1}^{*}=\Delta_{n}^{*} / \Delta_{n-1}$. The results in (18) are a consequence of these identities and (17).

The two dimensional sections $\mathfrak{R}_{2 n+1}$ for an $S$ sequence $\left\{c_{n}\right\}_{n=0}^{\infty}$ corresponding to $E_{n}$ are given by

$$
\left\{(x, y):\left(c_{0}, c_{1}, \cdots, c_{2 n-2}, x, y\right) \in \mathfrak{R}_{2 n+1}\right\} .
$$

If $\Delta_{n}>0, \Delta_{n, 1}>0$ for $n \leqq m$, these sections are the common part of the parabolic regions $E_{n}$ and the half plane $x \geqq \underline{c}_{2 n-1}$, where $\underline{c}_{2 n-1}$ is obtained from $\Delta_{n-1,1}$ by replacing $c_{2 n-1}$ with $c_{2 n-1}$ and setting the resulting determinant equal to zero. The coefficients in the $S$-fraction (8) are determined by

$$
d_{2 n}=\frac{\Delta_{n-2,1} \Delta_{n}}{\Delta_{n-1} \Delta_{n-1,1}}, d_{2 n+1}=\frac{\Delta_{n-1} \Delta_{n, 1}}{\Delta_{n-1,1} \Delta_{n}}(n=0,1,2, \cdots, m),
$$

where $\Delta_{-2,1}=\Delta_{-1,1}=\Delta_{-1}=1$ and $\left\{c_{n}\right\}_{n=0}^{\infty}$ is an $S$ sequence for which $\Delta_{n}>0, \Delta_{n-1,1}>0$ when $n \leqq m$.

THEOREM 4. Let $\left\{c_{n}\right\}_{n=0}^{\infty}$ be an $S$ sequence such that $\Delta_{n}>0, \Delta_{n-1,1}>0$ for $n \leqq m$. Then the coefficients in the $S$-fraction (8) corresponding to this sequence are determined by

$$
d_{2 n}=\frac{e_{n}}{\varepsilon_{n}}, d_{2 n+1}=\frac{\varepsilon_{n+1}}{e_{n}}
$$

where $e_{n}=c_{2 n}-\underline{c}_{2 n}, \varepsilon_{n}=c_{2 n-1}-\underline{c}_{2 n-1}, 0 \leqq n \leqq m$.

The result is a consequence of (19) and the identities $e_{n}=\Delta_{n} / \Delta_{n-1}$, $\varepsilon_{n}=\Delta_{n-1,1} / \Delta_{n-2,1}$.

\section{REFERENCES}

1. S. Barnard and J. M. Child, Higher Algebra, MacMillan, 1936.

2. C. Carathéodory, Über den Variabilitätsbereich der Koeffizienten von Potenzreihen, die Gegebene Werte Nicht Annehmen, Math. Ann., 64 (1907), 95-115.

3. H. Hamburger, Über eine Erweiterung des Stieltjesschen Momentenproblems, Math. Ann., 82 (1920), 168-187.

4. S. Karlin and L. S. Shapley, Geometry of moment spaces, Mem. Ämer. Math. Soc., 
no $12,1953$.

5. S. Karlin and W. J. Studden, Tchebycheff Systems: with Applications in Analysis and Statistics, Interscience, 1966.

6. M. G. Krein, The ideas of P. L. Čebyšev and A. A. Markov in the theory of limiting values of integrals and their further developments, Amer. Math. Soc. Trans. Ser. 2, 12 (1951), 1-122.

7. J. A. Shohat and J. D. Tamarkin, The problem of moments, Mathematical Surveys, no 1, Amer. Math. Soc., 1951.

8. H. S. Wall, Analytic Theory of Continued Fractions, Van Nostrand, 1948.

9. F. M. Wright, On the backward extension of positive definite Hamburger moment sequences, Proc. Amer. Math. Soc., 7 (1956), 413-422.

Received November 25, 1975.

UNIVERSITY OF CINCINNATI

AND

DENISON UNIVERSITY 



\section{PACIFIC JOURNAL OF MATHEMATICS}

\section{EDITORS}

RICHARD ARENS (Managing Editor)

University of California

Los Angeles, California 90024

\section{R. A. Beaumont}

University of Washington

Seattle, Washington 98105
J. DugundjI

Department of Mathematics

University of Southern California

Los Angeles, California 90007

D. Gilbarg and J. Milgram

Stanford University

Stanford, California 94305

\section{ASSOCIATE EDITORS}
E. F. BECKENBACH
B. H. NeumanN
F. WOLF
K. YosHIDA

\section{SUPPORTING INSTITUTIONS}

\author{
UNIVERSITY OF BRITISH COLUMBIA \\ CALIFORNIA INSTITUTE OF TECHNOLOGY \\ UNIVERSITY OF CALIFORNIA \\ MONTANA STATE UNIVERSITY \\ UNIVERSITY OF NEVADA \\ NEW MEXICO STATE UNIVERSITY \\ OREGON STATE UNIVERSITY \\ UNIVERSITY OF OREGON \\ OSAKA UNIVERSITY
}

\author{
UNIVERSITY OF SOUTHERN CALIFORNIA \\ STANFORD UNIVERSITY \\ UNIVERSITY OF HAWAII \\ UNIVERSITY OF TOKYO \\ UNIVERSITY OF UTAH \\ WASHINGTON STATE UNIVERSITY \\ UNIVERSITY OF WASHINGTON \\ AMERICAN MATHEMATICAL SOCIETY
}

The Supporting Institutions listed above contribute to the cost of publication of this Journal, but they are not owners or publishers and have no responsibility for its content or policies.

Mathematical papers intended for publication in the Pacific Journal of Mathematics should be in typed form or offset-reproduced, (not dittoed), double spaced with large margins. Please do not use built up fractions in the text of your manuscript. You may however, use them in the displayed equations. Underline Greek letters in red, German in green, and script in blue. The first paragraph or two must be capable of being used separately as a synopsis of the entire paper. Items of the bibliography should not be cited there unless absolutely necessary, in which case they must be identified by author and Journal, rather than by item number. Manuscripts, in triplicate, may be sent to any one of the editors. Please classify according to the scheme of Math. Reviews, Index to Vol. 39. All other communications should be addressed to the managing editor, or Elaine Barth, University of California, Los Angeles, California, 90024.

The Pacific Journal of Mathematics expects the author's institution to pay page charges, and reserves the right to delay publication for nonpayment of charges in case of financial emergency.

100 reprints are provided free for each article, only if page charges have been substantially paid. Additional copies may be obtained at cost in multiples of 50 .

The Pacific Journal of Mathematics is issued monthly as of January 1966. Regular subscription rate: $\$ 72.00$ a year (6 Vols., 12 issues). Special rate: $\$ 36.00$ a year to individual members of supporting institutions.

Subscriptions, orders for back numbers, and changes of address should be sent to Pacific Journal of Mathematics, 103 Highland Boulevard, Berkeley, California, 94708.

PUBLISHED BY PACIFIC JOURNAL OF MATHEMATICS, A NON-PROFIT CORPORATION

Printed at Kokusai Bunken Insatsusha (International Academic Printing Co., Ltd.), 8-8, 3-chome, Takadanobaba, Shinjuku-ku, Tokyo 160, Japan. 


\section{Pacific Journal of Mathematics \\ Vol. 65, No. $2 \quad$ October, 1976}

Andrew Adler, Weak homomorphisms and invariants: an example .......... 293

Howard Anton and William J. Pervin, Separation axioms and metric-like

functions ............................................. 299

Ron C. Blei, Sidon partitions and p-Sidon sets .................... 307

T. J. Cheatham and J. R. Smith, Regular and semisimple modules ........... 315

Charles Edward Cleaver, Packing spheres in Orlicz spaces .............. 325

Le Baron O. Ferguson and Michael D. Rusk, Korovkin sets for an operator on a

space of continuous functions ............................. 337

Rudolf Fritsch, An approximation theorem for maps into Kan fibrations ....... 347

David Sexton Gilliam, Geometry and the Radon-Nikodym theorem in strict

Mackey convergence spaces .................................

William Hery, Maximal ideals in algebras of topological algebra valued

functions ...................................... 365

Alan Hopenwasser, The radical of a reflexive operator algebra ........... 375

Bruno Kramm, A characterization of Riemann algebras................. 393

Peter K. F. Kuhfittig, Fixed points of locally contractive and nonexpansive

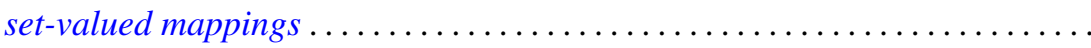

Stephen Allan McGrath, On almost everywhere convergence of Abel means of

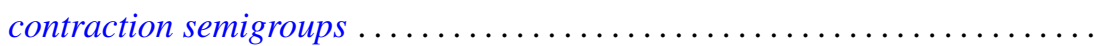

Edward Peter Merkes and Marion Wetzel, A geometric characterization of

indeterminate moment sequences............................ 409

John C. Morgan, II, The absolute Baire property ................... 421

Eli Aaron Passow and John A. Roulier, Negative theorems on generalized convex approximation .................................... 437

Louis Jackson Ratliff, Jr., A theorem on prime divisors of zero and characterizations of unmixed local domains ..............

Ellen Elizabeth Reed, A class of $T_{1}$-compactifications................... 471

Maxwell Alexander Rosenlicht, On Liouville's theory of elementary

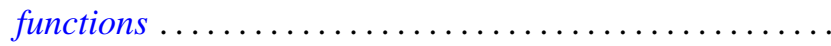

Arthur Argyle Sagle, Power-associative algebras and Riemannian

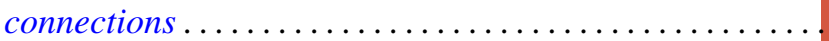

Chester Cornelius Seabury, On extending regular holomorphic maps from Stein manifolds...

Elias Sai Wan Shiu, Commutators and numerical ranges of powers of operators ...................................

Donald Mark Topkis, The structure of sublattices of the product of $n$ lattices ... 525

John Bason Wagoner, Delooping the continuous $K$-theory of a valuation

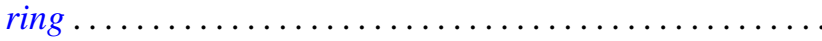

Ronson Joseph Warne, Standard regular semigroups...........

Anthony William Wickstead, The centraliser of $E \otimes_{\lambda} F \ldots$. 